\section{Matrix partitioning and trend assessment procedures in nonparametric analysis: BASIC programs}

\author{
G. N. WIGGANS and A. SAHGAL \\ MRC Neuroendocrinology Unit \\ Newcastle General Hospital \\ Newcastle upon Tyne, United Kingdom
}

There are several nonparametric tests for dealing with data that can be placed in contingency or in other tabular forms (e.g., Singer, 1979). Recently, several BASIC programs for microcomputers have been published that allow easy statistical analysis. However, most, if not all, require the repeated reentry of data even when the same table of scores has to be selectively reanalyzed. Consider, for example, the Friedman and Wilcoxon matched-pairs tests (Siegel, 1956). If the Friedman data matrix proves to be significant, indicating that certain conditions (columns) differ from others, then pairwise comparisons can be made using the Wilcoxon test; in most programs, the two tests have to be undertaken separately. Even more pertinent is the problem of partitioning a contingency table (Winer. 1971), in which selected treatments are compared against others; once again, most programs do not permit such procedures.

We describe two BASIC programs that deal with a number of these cases; in addition, a program to test for the significance of monotonic trends is also presented.

Program INFO. A powerful, but little known, nonparametric test, the information statistic (or likelihood ratio test), has been described for frequency data placed in $k$ nominal categories (Kullback, 1968; Robbins, 1977). Consider an example from passive avoidance, in which there are two groups of rats, one lesioned and the other sham operated, and each group is subdivided into salineor drug-injected conditions. Each subject's avoidance latency can be placed into one of a number of arbitrary categories: for example, Category I, number of animals with latencies of less than $30 \mathrm{sec}$, Category II, 30-60 sec, and Category III, greater than $60 \mathrm{sec}$. Thus, we have a matrix with four rows referring to the treatment conditions (sham-saline, sham-drug, lesion-saline, lesion-drug: Rows $1-4$, respectively) and three columns corresponding to latency categories. From this matrix of scores, we first establish whether the latency distributions (ratios) are significantly different, that is, whether there is significant "information" in the matrix. Only if this is the case may the following specific questions be asked: (1) Is there a

G. N. Wiggans is now at the Department of Zoology, University of Nottingham, University Park, Nottingham NG7 2RD, United Kingdom. A Sahgal's mailing address is: MRC Neuroendocrinology Unit, New castle General Hospital, Westgate Road, Newcastle upon Tyne. NE4 6BE, United Kingdom. lesion effect, regardless of drug, (2) is there a drug effect regardless of lesion. and (3) is there a drug $\times$ lesion interaction (i.e., does the drug affect lesioned animals differently from sham-operated animals)?

A statistic, termed $2 \hat{\mathrm{I}}$, can be computed by first calculating the expected frequency $(\mathrm{E})$ for each cell, where $\mathrm{E}=($ row total $\times$ column total) $/$ grand total. $2 \hat{\mathbf{I}}=2 \times$ Obs $\times \ln (\mathrm{Obs} / \mathrm{E})$ summed across all cells; Obs is the observed value in each cell, and in is the natural logarithm. $2 \hat{\mathrm{I}}$ is distributed as $\chi^{2}$ with $(\mathrm{r}-1) \times(\mathrm{k}-1)$ degrees of freedom (where $r$ and $k$ are the numbers of rows and columns, respectively). Specific treatment effects are computed by combining (collapsing) rows in a meaningful manner. In the example mentioned above, the lesion effect is derived by comparing Row $1+$ Row 2 with Row 3 + Row 4 ; the drug effect is derived by comparing Row 1 + Row 3 with Row 2 + Row 4; and the interaction effect is derived by subtracting both main effect 2Îs from the overall statistic. The degrees of freedom are treated similarly. The test can deal with any number of factors and, moreover, can be used to determine precisely which rows (treatments) differ from, for example, control scores. This would be useful in the case in which several doses had been given and we wished to determine which doses (if any) differed from control (e.g., Hulme, Sahgal, \& Iversen, 1979). The analysis bears some resemblance to the methods of partitioning a $\chi^{2}$ contingency table (Winer, 1971); however, it is more widely applicable, since fewer restrictions are imposed and small cell frequencies do not necessarily invalidate the analysis.

In addition to the partitioning procedure outlined above, the test (program) also permits any number of columns to be combined prior to analysis. This is particularly useful when we wish to collapse columns with low frequencies, or when extreme effects are to be investigated (e.g., Barnard, 1980; Sahgal \& Wright, 1983).

Program FWKM. Another illustration of partitioning comes from the Friedman and Kruskal-Wallis test for $k$ related and independent samples, respectively. If the relevant analysis is significant, selective comparisons may be made using an appropriate two-sample test (e.g., the Wilcoxon or the Mann-Whitney test). Although numerous programs exist for these four different tests, we present a single program that deals with related or independent samples and, moreover, permits partitioning without any further data entry.

Program JONCK. Finally, the statistical package contains a rather useful nonparametric trend test (Jonckheere, 1954). This analysis tests for positive or negative monotonic trends in data. For example, $m$ subjects may be tested in a conditioning experiment for $n$ days, with errors being recorded daily. The Jonckheere test permits an answer to the question of whether or not errors decrease over time, as would be expected if learning gradually occurred. 
Language and Programs. The three programs (INFO, FWKM, and JONCK) are written in enhanced BASIC as used by the British Broadcasting Corporation's (BBC) microcomputer, manufactured by Acorn Computers Ltd., Cambridge, United Kingdom. The programs can easily be altered for use in other BASIC machines; only standard commands and functions are used. Real numbers are stored to nine-figure accuracy, and the computed statistics are sufficiently accurate; the user need supply only the data, and an appropriate table of critical values for the statistic-ordinary $\chi^{2}$ tables are used for $2 \hat{\mathrm{I}}$.

Availability. Full listings of the programs, and sample printouts plus comments are available for a fee of $£ 4.00$, or approximately $\$ 5.00$ (money orders, in pounds sterling, made payable to the MRC Neuroendocrinology Unit), to cover costs.

\section{REFERENCES}

BARNARD, G. A. (1980). Note on an especially simple partitioning of chi-square from questionnaires. Psychological Bulletin, 87, 58.
Hulme, M., Sahgal, A., \& Iversen, S. D. (1979). Effects of sodium amylobarbitone on memory processes in the pigeon. Psychopharmacology, 62, 71-78.

JONCKHEERE, A. R. (1954). A test of significance for the relation between $m$ rankings and $k$ ranked categories. British Journal of Statistical Psychology, 7, 93-100.

Kullback, S. (1968). Information theory and statistics. New York: Dover.

Robiss, T. W. (1977). A critique of the methods available for the measurement of spontaneous motor activity. In L. L. Iversen, S. D. Iversen, \& S. H. Snyder (Eds.), Handbook of psychopharmacology (Vol. 7, pp. 37-82). New York: Plenum.

Sahgal, A., \& Wright, C. (1983). A comparison of the effects of vasopressin and oxytocin with amphetamine and chlordiazepoxide on passive avoidance behaviour in rats. Psychopharmacology, 80, 88-92.

SIEGEL, S. (1956). Nonparametric statistics for the behavioral sciences. New York: McGraw-Hill.

SINGER, B. (1979). Distribution-free methods for non-parametric problems: A classified and selected bibliography. British Journal of Mathematical and Statistical Psychology, 32, 1-60.

WINER, B. J. (1971). Statistical principles in experimental design. New York: McGraw-Hill.

(Revision accepted for publication June 15, 1984.) 